\title{
Magnetic dipole moments of ${ }^{58} \mathrm{Cu}$ and ${ }^{59} \mathrm{Cu}$ by in-source laser spectroscopy
}

\author{
N. J. Stone, ${ }^{1,2}$ U. Köster, ${ }^{3,4}$ J. Rikovska Stone,,${ }^{1,5}$ D. V. Fedorov,${ }^{6}$ V. N. Fedoseyev,${ }^{4}$ K. T. Flanagan, ${ }^{7}$ M. Hass, ${ }^{8}$ and S. Lakshmi ${ }^{8}$ \\ ${ }^{1}$ Department of Physics, University of Oxford, Oxford OXI 3PU, United Kingdom \\ ${ }^{2}$ Department of Physics and Astronomy, University of Tennessee, Knoxville, Tennessee 37996, USA \\ ${ }^{3}$ Institut Laue Langevin, 6 rue Jules Horowitz, F-38042 Grenoble Cedex 9, France \\ ${ }^{4}$ ISOLDE, CERN, CH-1211 Geneva 23, Switzerland \\ ${ }^{5}$ Department of Chemistry and Biochemistry, University of Maryland, College Park, Maryland 20742, USA \\ ${ }^{6}$ Petersburg Nuclear Physics Institute, RU-188350 Gatchina, Russia \\ ${ }^{7}$ Instituut voor Kern- en Stralingsfysica, K.U. Leuven, Celestijnenlaan 200D, B-3001 Leuven, Belgium \\ ${ }^{8}$ The Weizmann Institute of Science, Rehovot, Israel
}

(Received 20 December 2007; published 27 June 2008)

\begin{abstract}
Online measurements of the magnetic dipole moments and isotope shifts of ${ }^{58} \mathrm{Cu}$ and ${ }^{59} \mathrm{Cu}$ by the in-source laser spectroscopy method are reported. The results for the magnetic moments are $\mu\left({ }^{58} \mathrm{Cu}\right)=+0.52(8) \mu_{N}, \mu\left({ }^{59} \mathrm{Cu}\right)=$ $+1.84(3) \mu_{N}$ and for the isotope shifts $\delta v^{59,65}=1.72(22) \mathrm{GHz}$ and $\delta v^{58,65}=1.99(30) \mathrm{GHz}$ in the transition from the $3 d^{10} 4 s^{2} \mathrm{~S}_{1 / 2}$ ground state to the $3 d^{10} 4 p^{2} P_{1 / 2}$ state in $\mathrm{Cu}$. The magnetic moment of ${ }^{58} \mathrm{Cu}$ is discussed in the context of the strength of the subshell closure at ${ }^{56} \mathrm{Ni}$, additivity rules and large-scale shell model calculations.
\end{abstract}

DOI: 10.1103/PhysRevC.77.067302

PACS number(s): 21.10.Ky, 27.40.+z, 32.10.Fn, 32.80.Fb

Introduction. The magnetic dipole moments of the (predominantly) single $p_{3 / 2}$ proton ground states of light odd- $A$ $\mathrm{Cu}$ isotopes between ${ }^{57} \mathrm{Cu}(N=28)$ and ${ }^{69} \mathrm{Cu}(N=40)$ are the only examples of a complete set of measured moments for the same proton state spanning two major neutron closed shells. A full listing is given in Table I. The neutrons fill the $p_{3 / 2}, f_{7 / 2}$, and $p_{1 / 2}$ orbitals. With just one proton outside the closed shell at $Z=28$, the $\mathrm{Cu}$ isotopes close to neutron shell closure form a favoured system for sophisticated theoretical calculations which take the underlying nuclear structure as well established [1].

One topic of relevance to the study of nuclear moments in recent years has been the degree to which effects of meson exchange currents can be invoked to improve agreement between experiment and theory, in particular for ground states of nuclei having double closed shell $+/-$ one quasiparticle. For this theory to be truly tested the underlying single particle wave functions must be reliably known. These ideas were successfully applied to measured moments of ${ }^{69} \mathrm{Cu}$ and ${ }^{67} \mathrm{Ni}$, respectively, one neutron hole and one proton particle outside the ${ }^{68} \mathrm{Ni}$ core [2]. It was anticipated that the magnetic moment of ${ }^{57} \mathrm{Cu}$ would again be amenable to similar theoretical analysis, coupled with the moment of ${ }^{57} \mathrm{Ni}$, provided there is good shell closure at ${ }^{56} \mathrm{Ni}$.

Recent results on magnetic moments of ${ }^{59} \mathrm{Cu}$ and ${ }^{57} \mathrm{Cu}$ were quite surprising $[3,4]$. They showed strong deviations from predictions based upon the expected approach to a pure $p_{3 / 2}$ single proton quasiparticle configuration. The measured magnetic moment of the $I^{\pi}=3 / 2^{-}$ground state of ${ }^{57} \mathrm{Cu}$, although slightly higher than that of ${ }^{59} \mathrm{Cu}$, was lower than the moment of ${ }^{69} \mathrm{Cu}$ by close to 1 nuclear magneton. Furthermore, large-scale-shell-model (LSSM) calculations suggest that in the light odd- $A \mathrm{Cu}$ isotopes close to $N=28$, the $3 / 2^{-}$ ground state may remain mixed with less than $70 \%{ }^{56} \mathrm{Ni}$ closed-shell-based configuration [5,6]. Further experimental confirmation for the lack of subshell closure at $N=Z=28$ would be valuable.
The experimental result on ${ }^{57} \mathrm{Cu}$ comes from a wellestablished technique, $\beta \mathrm{NMR}$, however the observed resonance is narrow, consisting of one point which stands clearly below those at neighboring frequencies (see Fig. 1 Ref. [4]). It is important to seek additional evidence for the departure of nuclear moments in this region from the close-to-closed-shell predictions. This paper reports the search for such evidence through measurement of the magnetic moment of the ground state of the odd-odd isotope ${ }^{58} \mathrm{Cu}$, which, having the same proton configuration as ${ }^{57} \mathrm{Cu}$ and a single odd neutron outside the $N=28$ shell closure, is very sensitive to the magnetism of the proton configuration in ${ }^{57} \mathrm{Cu}$. The magnetic moment of odd-odd nuclei in such simple cases should be examples of basic additivity (neglecting the interaction between the odd valence nucleons) [7],

$$
\begin{aligned}
& g_{o-o}\left(I_{p} \otimes I_{n}=I\right) \\
& \quad=\frac{g_{p}+g_{n}}{2}-\frac{g_{p}-g_{n}}{2} \frac{I_{p}\left(I_{p}+1\right)-I_{n}\left(I_{n}+1\right)}{I(I+1)} .
\end{aligned}
$$

For the case of ${ }^{58} \mathrm{Cu}$ both odd proton and odd neutron have $I=3 / 2$, so the prediction is $g\left({ }^{58} \mathrm{Cu}\right)=\left[g\left({ }^{57} \mathrm{Cu}\right)+\right.$ $\left.g\left({ }^{57} \mathrm{Ni}\right)\right] / 2$. The magnetic moment of ${ }^{57} \mathrm{Ni}$ is $\mu\left({ }^{57} \mathrm{Ni}\right)=$ $-0.7975(14) \mu_{N}$ [8], thus $g_{n}=-0.5317$ (11). If the published value for the ${ }^{57} \mathrm{Cu} 3 / 2^{-}$ground state moment $\left(\mu\left({ }^{57} \mathrm{Cu}\right)=\right.$ $+2.00(5) \mu_{N}$ [4]), $g_{p}=+1.33(3)$ is taken, the predicted $g$-factor (and magnetic moment since $I=1$ ) for ${ }^{58} \mathrm{Cu}$ is $g_{o-o}=\mu\left({ }^{58} \mathrm{Cu}\right)=+0.40(2) \mu_{N}$. Taking as the ${ }^{57} \mathrm{Cu}$ moment the measured value for ${ }^{69} \mathrm{Cu}\left(\mu\left({ }^{69} \mathrm{Cu}\right)=+2.84(1) \mu_{N}\right.$ [3] $)$ leads to $g_{o-o}=\mu\left({ }^{58} \mathrm{Cu}\right)=+0.68(1) \mu_{N}$ The most recent shell model calculation of the ${ }^{57} \mathrm{Cu}$ moment is $2.490 \mu_{N}$ which, with simple additivity, gives for ${ }^{58} \mathrm{Cu}$ of $+0.564 \mu_{N}$, while the detailed calculation itself gives $+0.603 \mu_{N}$ [9]. An experimental result for the magnetic moment of ${ }^{58} \mathrm{Cu}$ has sensitivity to the moment of the odd proton state, and, in turn, to details of its wave function. 
TABLE I. Experimental values of ground state magnetic moments of $\mathrm{Cu}$ isotopes from ${ }^{57} \mathrm{Cu}$ to ${ }^{69} \mathrm{Cu}[3,4,8,11,15]$ and this experiment $\left({ }^{58} \mathrm{Cu}\right)$. Theoretical values, calculated with empirical single-particle $g$-factors $g_{l}^{p}=1.1, g_{l}^{n}=-0.1$, and $g_{s}=0.9 g_{s}^{\text {free }}$ in LSSM, are from [5,9].

\begin{tabular}{lcccc}
\hline \hline Mass & $N$ & $I^{\pi}$ & \multicolumn{1}{c}{$\mu_{\exp }\left(\mu_{N}\right)$} & $\mu_{\text {th }}\left(\mu_{N}\right)$ \\
\hline 57 & 28 & $3 / 2-$ & $2.00(5)$ & +2.489 \\
59 & 30 & $3 / 2-$ & $+1.891(9)$ & +1.886 \\
61 & 32 & $3 / 2-$ & $+2.14(4)$ & +2.193 \\
63 & 34 & $3 / 2-$ & $+2.2273456(14)$ & +2.251 \\
65 & 36 & $3 / 2-$ & $+2.38161(19)$ & +2.398 \\
67 & 38 & $3 / 2-$ & $+2.54(2)$ & +2.545 \\
69 & 40 & $3 / 2-$ & $+2.84(1)$ & $+2.853^{\mathrm{a}}$ \\
58 & 29 & $1+$ & $+0.52(8)$ & +0.60 \\
60 & 31 & $2+$ & $+1.219(3)$ & +1.159 \\
62 & 33 & $1+$ & $-0.380(4)$ & -0.236 \\
64 & 35 & $1+$ & $-0.271(2)$ & -0.114 \\
66 & 37 & $1+$ & $-0.282(2)$ & +0.490 \\
68 & 39 & $1+$ & $+2.55(19)$ & $+2.25^{\mathrm{b}}$ \\
\hline \hline
\end{tabular}

${ }^{\mathrm{a}}$ From [2].

${ }^{\mathrm{b}}$ From [15].

Experiment, Data Analysis, and Results. The experiment was carried out at the ISOLDE isotope separator facility, CERN, Geneva using the in-source laser spectroscopy method described fully in Refs. [10,11]. The pulsed beam of $1.4 \mathrm{GeV}$ protons was incident upon an ISOLDE $\mathrm{ZrO}_{2}$ fiber target [12]. Radioisotopes produced by spallation diffused from the target to the ca. $2000^{\circ} \mathrm{C}$ hot ionizer cavity. Two laser beams at $327.4 \mathrm{~nm}$ and $287.9 \mathrm{~nm}$ wavelength interacted with $\mathrm{Cu}$ atoms in the source to excite them from the $3 d^{10} 4 s^{2} S_{1 / 2}$ ground state via the intermediate $3 d^{10} 4 p^{2} P_{1 / 2}$ state to the $3 d^{9} 4 s 5 s^{2} D_{3 / 2}$ auto-ionizing state, producing ions which were subsequently accelerated to $60 \mathrm{keV}$ and mass separated. The first step excitation used a narrow-band laser which could be tuned to explore the hyperfine structure of the ground and intermediate states. The mass-separated ions were implanted into a tape and transported rapidly to a $4 \pi$ beta detection station where the ion beam intensity was measured as a function of the narrow-band laser frequency. Simultaneously a current of stable ${ }^{63} \mathrm{Cu}$ ions generated in the same ion source system was measured to monitor the laser power and Doppler broadening and check for other systematic variations.

In both the upper and lower levels of the first step excitation the nuclear spin $I$ (here taken as $I=1$ appropriate for ${ }^{58} \mathrm{Cu}$ ) couples to the electron spin $J=1 / 2$ to give a hyperfine split doublet, with total spin $F=3 / 2,1 / 2$. The splitting is given by [1]

$$
E_{F}=A[F(F+1)-I(I+1)-J(J+1)] / 2,
$$

where $A$ is the magnetic hyperfine interaction strength for the particular ionic state. There are four allowed transitions with $\Delta F= \pm 1,0$ in the resonance spectrum, however limitations in the resolution of the system, associated with Doppler width in the hot cavity of the ion source and also with power broadening caused by saturation of the $327.4 \mathrm{~nm}$ laser transition, mean that the components may not be resolved. The $A$ parameters for the ${ }^{2} P_{1 / 2}$ and ${ }^{2} S_{1 / 2}$ states are related, having an isotopeindependent ratio of $+0.086[13,14]$, thus a single magnetic hyperfine interaction parameter, $A^{2} S_{1 / 2}$, is fitted [10].

Analysis of the data on the ground states of stable ${ }^{63} \mathrm{Cu}$ $\left(I^{\pi}=3 / 2^{-}\right)$and radioactive ${ }^{58} \mathrm{Cu}\left(I^{\pi}=1^{+}, T_{1 / 2}=3.20 \mathrm{~s}\right)$ and ${ }^{59} \mathrm{Cu}\left(I^{\pi}=3 / 2^{-}, T_{1 / 2}=81.5 \mathrm{~s}\right)$ was made using the programme of Gheysen et al. which is fully described in [15]. As well as setting the nuclear and electronic spins of the isotope and electronic state under study, there are adjustable parameters to allow for Doppler width and laser power broadening. The variables of nuclear structure interest are the magnetic hyperfine interaction parameter $A$ and the isotope shift $S$. For the electronic states involved the electric quadrupole interaction is zero as both have spin $J=1 / 2$.

The data on ${ }^{63} \mathrm{Cu}$ were used to establish the parameters of the laser system affecting the power broadening and the Gaussian width to be adopted in fitting the hyperfine interactions of the other, radioactive, isotope for which data were taken during the same scan. Although the absolute laser frequency is not precisely determined in the setup, the fitting routine (see below) determined the isotope shift for each nucleus, relative to a nominal frequency of $30535.3 \mathrm{~cm}^{-1}$. Since the isotope shift of ${ }^{63} \mathrm{Cu}$ relative to ${ }^{65} \mathrm{Cu}$ is known for this electronic transition it is possible to extract isotope shifts for the two radioactive isotopes also, relative to ${ }^{65} \mathrm{Cu}$. The magnetic hyperfine interaction parameter $A$ of ${ }^{63} \mathrm{Cu}$ was also obtained by fitting to act as a test of the analysis by comparing with the known value $A\left({ }^{63} \mathrm{Cu}\right)=5.866908706(20) \mathrm{GHz}$ [13].

The magnetic moments of ${ }^{59} \mathrm{Cu}$ and ${ }^{58} \mathrm{Cu}$. The results of fitting three scans taken on ${ }^{63} \mathrm{Cu}$ are given in Table II. The values for $A$ are all in close agreement with the established value given above. Of the three scans the first was made simultaneously with taking data for ${ }^{59} \mathrm{Cu}$. The ionization rate $\Gamma$ and Gaussian width $G$ fitted to the ${ }^{63} \mathrm{Cu}$ data were adopted in fitting the ${ }^{59} \mathrm{Cu}$ data for $A$ and $S$, yielding the values given in Table II. The Gaussian width used in the fitting was in all cases between 4.3 and $4.7 \mathrm{GHz}$, broadly compatible with the value 4.9(3) GHz used by Gheysen et al. [15]. This Gaussian width is composed of the Doppler width in the ca. $2000^{\circ} \mathrm{C}$ hot cavity (ca. $4 \mathrm{GHz}$ ) and the bandwidth of the frequency-doubled dye laser beam (ca. 1.2 GHz before doubling). The magnetic

TABLE II. Experimental fitting results for the magnetic interaction strength $A$ and isotope shift $S$. The laser ionization rate $\Gamma$ and the Gaussian width $G$ are also shown. The magnetic moment was evaluated for fully completed scans only, other mass 63 scans were used to set laser and ion source parameters.

\begin{tabular}{lclllll}
\hline \hline Mass Scan & $\begin{array}{c}A\left(\chi_{\min }^{2}\right) \\
(\mathrm{GHz})\end{array}$ & $\begin{array}{c}\mu_{\exp } \\
\left(\mu_{N}\right)\end{array}$ & $\begin{array}{c}\Gamma \\
(\mathrm{GHz})\end{array}$ & $\begin{array}{c}G \\
(\mathrm{GHz})\end{array}$ & $\begin{array}{c}S\left(\chi_{\min }^{2}\right) \\
(\mathrm{GHz})\end{array}$ \\
\hline 63 & 1 & $5.84_{-0.21}^{+0.18}$ & $2.22(9)$ & 0.27 & $4.7(1)$ & 0.0000 \\
63 & 2 & 5.87 & & 0.21 & $4.3(3)$ & 0.0000 \\
63 & 3 & 5.86 & & 0.20 & $4.6(1)$ & 0.0000 \\
59 & 1 & $4.87_{-0.07}^{+0.09}$ & $1.84(3)$ & 0.27 & $4.7(1)$ & $1.14(21)$ \\
58 & 2 & $2.38_{-0.62}^{+0.38}$ & $0.54(14)$ & 0.21 & $4.3(3)$ & $1.45(41)$ \\
58 & 3 & $1.98_{-0.30}^{+0.40}$ & $0.51(9)$ & 0.20 & $4.6(1)$ & $1.36(41)$ \\
\hline \hline
\end{tabular}




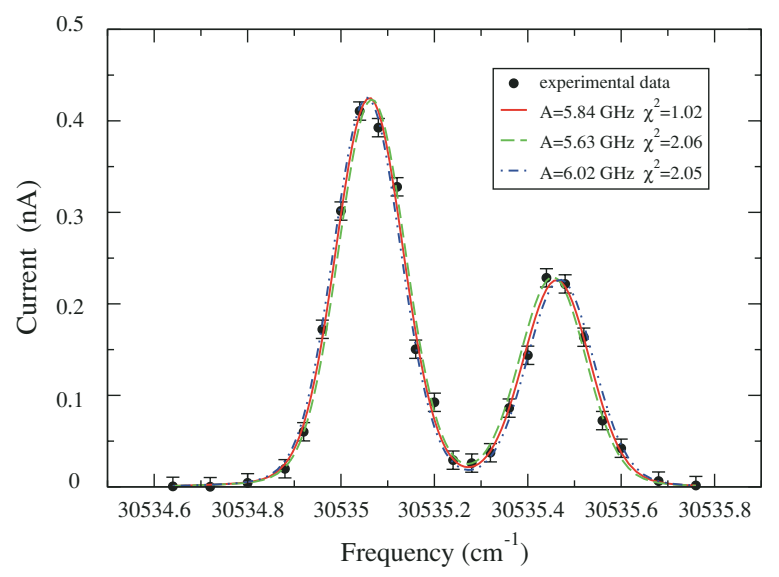

FIG. 1. (Color online) Fitting to data of ${ }^{63} \mathrm{Cu}$ scan 1 . The curves shown are best fit and upper and lower limits for the magnetic interaction strength $A$. The isotope shift was kept constant.

moment result, $+1.84(3) \mu_{N}$ is in good agreement with the more precise NMR/ON value $+1.891(9) \mu_{N}$ of Golovko et al . and formed a guide for their resonance search [3].

The second and third scans on ${ }^{63} \mathrm{Cu}$ were taken in conjunction with ${ }^{58} \mathrm{Cu}$ data during two separate experiments. The fitted A parameters for ${ }^{63} \mathrm{Cu}$ are both in good agreement with the established value, as expected. As can be seen in Figs. 1 to 3 the frequency range needed to cover the full resonance of ${ }^{58} \mathrm{Cu}$ is considerably narrower than for the ${ }^{63} \mathrm{Cu}$ and ${ }^{59} \mathrm{Cu}$ resonances, thus the data on ${ }^{63} \mathrm{Cu}$ taken during the ${ }^{58} \mathrm{Cu}$ scans were less complete than those taken during the ${ }^{59} \mathrm{Cu}$ scan and no detailed ranges of fit for the $A$ and $S$ parameters for ${ }^{63} \mathrm{Cu}$ were calculated from these data. The ${ }^{58} \mathrm{Cu}$ scans showed that the resonance transitions were barely resolved and that this isotope presents a major challenge to the method. Nevertheless, adopting the laser settings given by the simultaneous ${ }^{63} \mathrm{Cu}$ fits, fits to the ${ }^{58} \mathrm{Cu}$ data gave consistent results, if with rather disappointingly wide uncertainty limits, with $A$ values of $2.4_{-0.6}^{+0.4} \mathrm{GHz}$ and $2.0_{-0.3}^{+0.4} \mathrm{GHz}$, respectively (see Table II). The error limit calculation for the second ${ }^{58} \mathrm{Cu}$ scan is shown in Fig. 4. The weighted average value for the

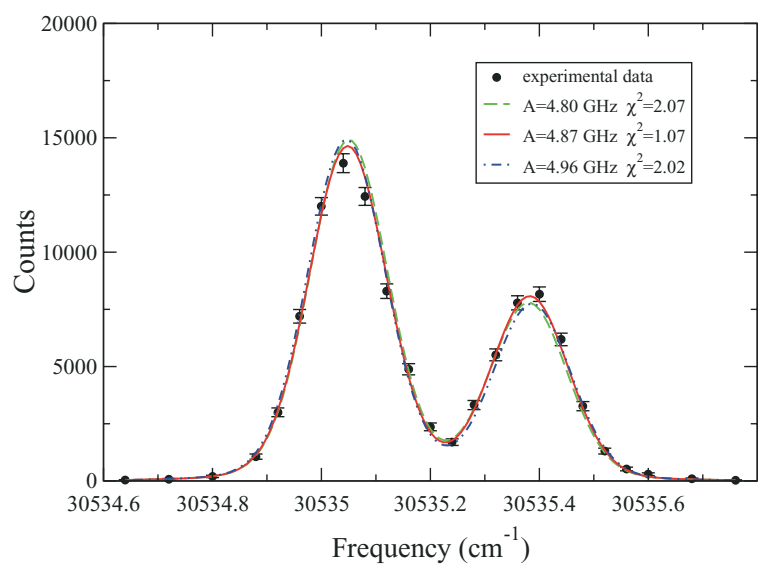

FIG. 2. (Color online) Fitting to data of ${ }^{59} \mathrm{Cu}$ scan 1 . The curves shown are best fit and upper and lower limits for the magnetic interaction strength $A$ with constant isotope shift.

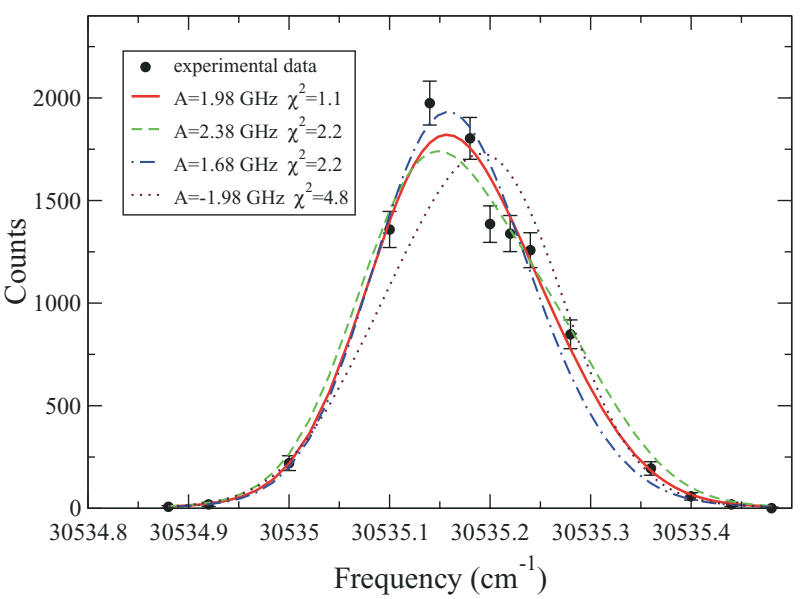

FIG. 3. (Color online) Fitting to data of ${ }^{58} \mathrm{Cu}$ scan 3. The curves shown are best fit and upper and lower limits for the magnetic hyperfine interaction strength $A$ and a constant isotope shift. Also shown is a calculated curve for A negative, at the best fit value, to establish the sign of the interaction.

magnetic dipole moment of ${ }^{58} \mathrm{Cu}$ is

$$
\mu\left({ }^{58} \mathrm{Cu}\right)=+0.52(8) \mu_{N} .
$$

The fits of the second scan are shown in Fig. 4, which also includes a calculation for a negative moment to demonstrate that the sign is well determined from the data.

The isotope shifts for ${ }^{59} \mathrm{Cu}$ and ${ }^{58} \mathrm{Cu}$. The isotope shifts of ${ }^{58,59} \mathrm{Cu}$ respectively were obtained from the displacement of the center-of-mass frequency of their hyperfine-split resonance with respect to the simultaneously measured ${ }^{63} \mathrm{Cu}$. The relative shift $\delta v^{59,63}$ is $1.14(21) \mathrm{GHz}$ and $\delta v^{58,63}$ has the average value $1.41(29) \mathrm{GHz}$. Adding these to the value $\delta v^{63,65}=0.58(8)$ $\mathrm{GHz}$ (weighted average of Refs. [16,17]) yields the isotope shifts relative to ${ }^{65} \mathrm{Cu}$ as

$\delta v^{59,65}=1.72(22) \mathrm{GHz} \quad$ and $\quad \delta v^{58,65}=1.99(30) \mathrm{GHz}$.

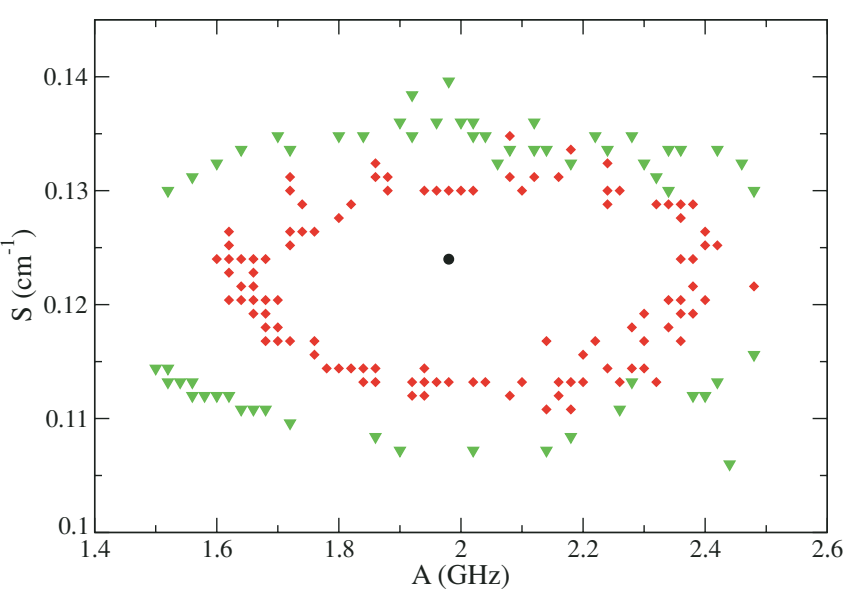

FIG. 4. (Color online) A matrix of reduced $\chi^{2}$ values for calculated curves for a range of parameters $A$ and $S$ showing the best fit (solid circle), the locus of $\chi_{\min }^{2}$ plus 1 (diamonds) and $\chi_{\min }^{2}$ plus 2 (triangles). 
Discussion and Conclusions. These experiments further demonstrate the value of the in-source laser spectroscopy method to give useful results for magnetic moments and isotope shifts of low-yield radioactive isotopes. The partial resolution of the multiplet transitions, manifest most clearly in the case of ${ }^{58} \mathrm{Cu}$, places unavoidable limits on the precision that can be achieved. The result for the magnetic moment of this isotope lies between the simple-additivity predictions +0.40 (2) $\mu_{N}$, based on the experimental odd- $A$ moments of its relevant neighbors and $+0.68(1) \mu_{N}$, calculated taking the ${ }^{57} \mathrm{Cu}$ equal to the moment ${ }^{69} \mathrm{Cu}$ (assuming symmetry in the $N=28-40$ neutron shell). No clear preference between the two limits is established. Although lower than the recent LSSM prediction (which assumes ${ }^{48} \mathrm{Ca}$ core), the result shows a similar degree of agreement as other odd-odd spin 1 isotopes (see Table I) with LSSM.

For mirror nuclei, the isoscalar and isovector components of a magnetic dipole moment $\mu$ can be written as [18]

$$
\mu^{\mathrm{IS} / \mathrm{IV}}=\left[\mu\left(T=+T_{\mathrm{z}}\right) \pm \mu\left(T=-T_{\mathrm{z}}\right)\right] / 2,
$$

where $T, T_{\mathrm{z}}$ is the isospin and its $z$-component. For selfconjugate $N=Z$ nuclei the isovector component vanishes and the isoscalar part can be expressed in terms of the spin $I$

$$
I=\left\langle i\left|S_{z}\right| i\right\rangle_{M=J}+\left\langle i\left|L_{z}\right| i\right\rangle_{M=J}
$$

as

$$
\left[\mu^{\mathrm{IS}}-I / 2\right] / 0.380=\left\langle i\left|S_{\mathrm{Z}}\right| i\right\rangle_{M=J}+\text { corr, }
$$

where the factor 0.380 is the difference between free nucleon isoscalar spin and orbital $g$-factors, $g_{s}^{\mathrm{IS}}=0.880$ and $g_{l}^{\mathrm{IS}}=0.5$. 'corr' stands for shell-dependent correction terms which are expected to be small [18]. As in our case $\mu^{\mathrm{IS}}$ equals $\mu^{\exp }$ and $I=1$, application of Eq. (7) indicates a small total (isoscalar) spin contribution to the magnetic dipole moment. The present result provides valuable guidance for possible future more precise experiments.

The isotope shift values are consistent with normal decrease of nuclear radii with falling mass number.

This work has been supported by the U.S. DOE grants DE-FG02-96ER40983 (UT), DE-FG02-94ER40834 (UMD), the European Union Sixth Framework through R113EURONS (contract no. 506065) and the Israel Science Foundation.
[1] B. Castel and I. S. Towner, Modern Theories of Nuclear Moments (Clarendon Press, Oxford, 1990).

[2] J. Rikovska et al., Phys. Rev. Lett. 85, 1392 (2000).

[3] V. V. Golovko et al., Phys. Rev. C 70, 014312 (2004).

[4] K. Minamisono et al., Phys. Rev. Lett. 96, 102501 (2006).

[5] M. Honma, T. Otsuka, B. A. Brown, and T. Mizusaki, Phys. Rev. C 69, 034335 (2004).

[6] A. F. Lisetskiy et al., Phys. Rev. C 68, 034316 (2003).

[7] P. C. Zalm, J. F. A. von Hienen, and P. W. Glaudemans, Z. Phys. A 287, 255 (1978).

[8] N. J. Stone, At. Data Nucl. Data Tables 90, 75 (2005).

[9] M. Honma, private communication, 2007.

[10] U. Köster et al., Nucl. Instrum. Methods B 160, 528 (2000).
[11] L. Weissman et al., Phys. Rev. C 65, 024315 (2002).

[12] U. Köster et al., Nucl. Instrum. Methods B 204, 303 (2003).

[13] H. Figger, D. Schmitt, and S. Penselin, in 'La Structure Hyperfine Magnétique des Atomes et des Molécules', Colloques Internationaux du CNRS, Paris, No. 164, 355 (1967).

[14] H. Bergström, W. X. Peng, and A. Persson, Z. Phys. D 13, 203 (1989).

[15] S. Gheysen, G. Neyens, and J. Odeurs, Phys. Rev. C 69, 064310 (2004).

[16] P. Brix and W. Humbach, Z. Phys. 128, 506 (1950).

[17] Marburger Sitzungsberichte 75, 5 (1952).

[18] B. A. Brown and B. H. Wildenthal, Phys. Rev. C 28, 2397 (1983). 